\title{
REAL INFLECTIONS OF HINGED PLANAR FOUR-BAR COUPLER CURVES
}

\author{
P.S.Donelan \& C.P.SCOTT \\ Victoria University of Wellington, New Zealand
}

20th May 1994

\begin{abstract}
Plücker's and Klein's equations provide an upper bound on the number of real inflections on the coupler curve of a hinged planar four-bar mechanism. Generally, for any configuration of the four-bar, the coupler points whose trajectories exhibit inflections lie on a circle. The coupler plane is partitioned by the envelope of the inflection circles into connected regions within which every coupler point has the same number of inflections on its trajectory. This enables us to locate coupler curves exhibiting the maximum possible number of inflections.
\end{abstract}

\section{$\S 1$ INTRODUCTION}

Four-bar mechanisms provide a rich source of geometrical problems, some of considerable complexity. The problem of finding design parameters for which some coupler curve exhibits particular features dates back at least as far as James Watt. In his case the desire was to find a mechanism with approximate straight-line output and since that time many refinements and improvements have been found for this problem. To a considerable extent, this has depended on locating ordinary and higher inflections on coupler curves, though it must be said that this in itself is not sufficient for good straight-line approximation over a reasonable range of input angles - the approximation may be valid only very close to the inflection.

One outstanding problem is to determine the maximum number of (real) inflection points that may be found on a single coupler curve. Here we seek to answer that question for hinged planar four-bar mechanisms (4R mechanisms), hereafter referred to simply is fourbar mechanisms. In theory this is a problem of algebraic geometry. That four-bar coupler curves are algebraic curves of degree six was discovered by Roberts [13]. Regarding them as complex projective curves and using standard results on their singularities, applications of

1991 Mathematics Subject Classification. Primary: 70B15, secondary: 14Q05, 53A17.

Key words and phrases. Four-bar, coupler curve, inflection.

Typeset by $\mathcal{A} \mathcal{M} \mathcal{S}-\mathrm{T}_{\mathrm{E}} \mathrm{X}$ 
Bézout's Theorem and Plücker's equations yield the precise number of complex inflections. Since the equations are in fact real algebraic, Klein's equation may also be used and this in turn gives an upper bound on the number of real inflections. However, attempting to enumerate the exact number requires one to find the simultaneous real solutions of the algebraic equation with its Hessian. Even after accounting for known double points the resultant has degree 32 and the task is too complicated in general.

On the other hand one may also employ kinematic geometry to locate inflections: at (almost) any configuration of the four-bar the coupler points whose curves exhibit inflections lie on a circle, so in principle the number of inflections on the curve can be found by counting the number of inflection circles which pass through the coupler point over the whole set of configurations of the four-bar. While this is also impractical, nevertheless the envelope of the inflection circles partitions the coupler plane into regions within which this number is constant. Pictures of this partition are then constructed using computer graphic routines and a search through the regions yields coupler curves with any (even) number of real inflections up to the maximum allowed by Klein's equation. The number can be confirmed by counting sign changes in the curvature function along the coupler curve. The approach employed here is in the spirit of the programme for classifying coupler curves established by Reinhold Müller [10,11].

Section 2 presents a brief account of the necessary information concerning four-bars. In section 3 the theoretical maximum number of real inflections is obtained by means of algebraic geometry applied to the equation for the coupler curve. The kinematic geometry used to partition the coupler plane is given in section 4. Results of the search, including computer-generated coupler curves exhibiting the maximum number of inflections appear in section 5 .

The results in this paper form part of the M.Sc. thesis of the second author. The authors are indebted to Dr. Chris Gibson of Liverpool University for first suggesting the problem of enumerating real inflections for four-bars and to Dr. Colin Bailey of Victoria University who created the 'FloatingPictures' drawing environment within which the graphics programming was created.

\section{$\S 2$ Four-Bar MeCHANisms}

The design parameters for the four-bar linkage are the lengths $d_{1}, d_{2}, d_{3}, d_{4}$ of the four bars, where $d_{4}$ is taken to be the length of the base and $d_{2}$ that of the coupler bar. Following Darboux, the configuration space of the mechanism can be described in terms of unit vectors $\left(x_{i}, y_{i}\right), i=1,2,3$ along the corresponding bars (see, for example Gibson and Newstead [5]). The six variables are connected by five equations, expressing the fact 
that each vector has unit length and that the four-bar forms a closed quadrilateral. By regarding the variables together with an extra one, $w$, as homogeneous coordinates in $P \mathbb{C}^{6}$ we are led to the complex projective variety given by:

$$
\begin{aligned}
x_{i}^{2}+y_{i}^{2} & =w^{2}, \quad i=1,2,3 \\
d_{1} x_{1}+d_{2} x_{2}+d_{3} x_{3} & =d_{4} w \\
d_{1} y_{1}+d_{2} y_{2}+d_{3} y_{3} & =0 .
\end{aligned}
$$

While this variety has degree eight, it contains two lines at infinity, each of which the residual curve of degree six meets twice. It can be shown that this residual variety has no singular points unless the Grashof condition

$$
d_{1} \pm d_{2} \pm d_{3} \pm d_{4}=0
$$

is satisfied. Four-bars for which (2) does not hold shall be called generic. Those that satisfy (2) are classified in [5] according to their singularities or equivalently by the geometry of the quadrilateral formed by the four-bar. The classification can be visualised in terms of the equations (2). In the positive octant of the four-dimensional space of design parameters, the equations define eight hypersurfaces, of which one (all signs positive) does not correspond to realisable mechanisms. Four of the others (with one negative) define rigid four-bars and thus determine boundaries of the region of realisable four-bars. The remaining three intersect in pairs in three planes and mutually in the line $d_{1}=d_{2}=d_{3}=d_{4}$. The hypersurfaces are the circumscriptible four-bars. Two of the planes correspond to kite configurations and one to parallelograms while the common line represents the rhombuses.

The equations (1), with $w=1$, may be used to define the configuration space as a real algebraic variety in $\mathbb{R}^{6}$. Write the design parameters in non-decreasing order of magnitude, when they shall be donoted $e_{1}, \ldots, e_{4}$. Let $E=e_{1}+e_{4}-e_{2}-e_{3}$ denote the sum of the shortest and longest sides minus the lengths of the other two. Then it is a standard fact (again, derived in [5]) that for $E<0$ the configuration space is a one-dimensional manifold consisting of two connected components (topologically circles), while if $E>0$ the manifold has only one component. An invaluable refinement is the classification of generic four-bars, described by Hain [6], in which they are described by means of the rocking (R) or cranking $(\mathrm{C})$ capabilities of the input/output bars and in particular whether they rock inwards (i) or outwards (o). This is presented in Table 1. Indeed these eight classes are the connected components of the complement of the three hyperplanes in the space of design parameters, mentioned above. They are pictured in Figure 1 in which the parameters are scaled by setting $d_{4}=1$. This illustrates the classifications of both generic and non-generic mechanisms. 
Table 1. Hain Classification

\begin{tabular}{llll}
\hline Hain class & description & \multicolumn{2}{c}{ Recognition principle } \\
\hline $\mathrm{R}_{\mathrm{io}}$ & double rocker & $E>0$ & $e_{4}=d_{1}$ \\
$\mathrm{R}_{\mathrm{oo}}$ & & & $e_{4}=d_{2}$ \\
$\mathrm{R}_{\mathrm{oi}}$ & & & $e_{4}=d_{3}$ \\
$\mathrm{R}_{\mathrm{ii}}$ & & $e_{4}=d_{4}$ \\
\hline $\mathrm{CR}_{1}$ & crank-rocker & $E<0$ & $e_{1}=d_{1}$ \\
$\mathrm{DR}$ & double crank & & $e_{1}=d_{2}$ \\
$\mathrm{CR}$ & crank-rocker & & $e_{1}=d_{3}$ \\
$\mathrm{DL}$ & double crank & & $e_{1}=d_{4}$ \\
\hline
\end{tabular}

\section{Figure 1. Diagram of the Classification of Four-Bars}

Our interest is in the coupler curves generated by four-bars. The Roberts Triple Generation Theorem [13] ensures that any coupler curve of a four-bar is also a coupler curve of two cognate mechanisms. Up to a scaling factor these cognates are realised by a cyclic permutation of the design parameters $d_{1}, d_{2}, d_{3}$ and thus corresponds to the cyclic permutation of axes in Figure 1. As a result, from the point of view of generation of coupler curves, the eight classes of Hain in Table 1 may be grouped into four cognate classes, denoted by Marsh [8]:

$$
\mathrm{I}: \mathrm{R}_{i o}, \mathrm{R}_{o o}, \mathrm{R}_{o i} ; \quad \mathrm{I}^{\prime}: \mathrm{R}_{i i} ; \quad \mathrm{II}: \mathrm{CR}_{1}, \mathrm{DR}, \mathrm{CR}_{2} ; \quad \mathrm{II}^{\prime}: \mathrm{DL} .
$$

\section{$\S 3$ Algebraic Geometry of the Coupler Curve}

We first recap briefly on the known algebraic geometry of four-bar coupler curves. The equation for a coupler curve of a generic four-bar, as derived by Roberts, takes the following form (see, for example, Hunt [7]):

$$
F \equiv u^{2}+v^{2}-w^{2}=0
$$

where $u(x, y), v(x, y)$ are polynomials of degree three and $w(x, y)$ is quadratic. Thus the coupler curve has degree six. The conic $w=0$ is a circle and $F(x, y)$ has a singularity if and only if $(x, y)$ lies on this circle, the circle of singular foci. As a complex projective curve, the coupler has triple points at each of the isotropic points $(1, \pm i, 0) \in P \mathbb{C}^{2}$. This then accounts for six intersections of the coupler curve with the circle of singular foci, which must be 12 in all by Bézout's Theorem. Since every other intersection is also a singular 
point, it must have multiplicity $\geq 2$. Thus there are at most three finite double points and cusps on the coupler curve. For a curve of degree $n$ there are at most $\frac{1}{2}(n-1)(n-2)$ double points, and hence 10 for a curve of degree six. Since there are in fact nine (each triple point counting as three double points) the deficit from the maximum, which is the genus of the curve, is one.

In fact for any coupler point (except the ends of the coupler bar) the complex coupler curve is a projection of the complex residual curve in $P \mathbb{C}^{6}$ described in section 2 and so has degree six (Gibson and Newstead [5]). The coupler curve has the same geometric genus as the corresponding residual curve, that is to say it is elliptic (genus 1) for generic four-bars (as we have already said) and rational (genus 0) for Grashof four-bars.

Plücker's equations, whose derivation can be found, for example, in Coolidge [2], now enable us to deduce further information concerning the characteristics of the curve. Let $n$ be the degree of a complex projective curve, $m$ its class, $\delta$ the number of double points (counting multiplicities), $\kappa$ the number of cusps, $\iota$ the number of inflections and $\tau$ the number of bitangents. Then

$$
\begin{gathered}
m=n(n-1)-2 \delta-3 \kappa \\
\iota=3 n(n-2)-6 \delta-8 \kappa
\end{gathered}
$$

For a coupler curve of a generic four-bar without cusps (so $\delta=9$ ) the class $m=30-18=$ 12. Marsh [8] shows that all cusps are real and only coupler points on the moving centrode (see section 4 below) possess cusps so that for points off this curve the above holds. The presence of a cusp reduces the class by one. From the second equation we can deduce that $\iota=72-54=18$ in the case of no cusps and this number reduces by two for each cusp on the coupler curve. For non-generic four-bars, for which $\delta \leq 10$, we have $m \leq 10$ and $\iota \leq 12$.

All of the above concerns complex characteristics of the curve. It has already been noted that cusps may only be real. We now wish to determine how many of the inflections may be real. Since the coupler curve is in fact real algebraic and the real singularities of the curve are no worse than ordinary double points and cusps, Klein's equation applies [2]. We must first distinguish among the complex Plücker characteristics as follows: let $\iota^{\prime}$ denote the number of real inflections, $\kappa^{\prime}$ the number of real cusps, $\delta_{2}^{\prime}$ the number of conjugate double points (ie acnodes) and $\tau_{2}^{\prime}$ the number of conjugate bitangents (ie real bitangents whose points of tangency with the curve are themselves complex conjugate points). Then Klein's equation can be written:

$$
n+2 \tau_{2}^{\prime}+\iota^{\prime}=m+2 \delta_{2}^{\prime}+\kappa^{\prime} .
$$


For a generic four-bar and a coupler curve without cusps, it follows that

$$
\iota^{\prime}=6+2\left(\delta_{2}^{\prime}-\tau_{2}^{\prime}\right) .
$$

Moreover since the acnodes occur among the three finite double points, $0 \leq \delta_{2}^{\prime} \leq 3$ and hence

$$
\iota^{\prime} \leq 12 \text {. }
$$

For mobile non-generic four-bars $m-n \leq 4$ but the number of finite double points is four. However among these must be at least one crunode arising from the singular configuration(s) of the four-bar. Hence again $0 \leq \delta_{2}^{\prime} \leq 3$ and so $\iota^{\prime} \leq 10$.

It is worth noting that while acnodes are real (isolated) points of the algebraic coupler curve, they do not correspond to actual configurations of the mechanism, which for nontrivial mechanisms must lie on analytic curves. The actual set of configurations thus forms what is known as a semi-algebraic set.

A necessary condition for an inflection on an algebraic curve is that the Hessian of the curve must vanish at the point. This is the source of the second Plücker equation abovethe Hessian and the original curve also intersect at singularities, with multiplicity 6 at a double point and 8 at a cusp. In principle, the location of the real inflections could be achieved by finding the resultant of the two equations and looking for real roots. However, even after factoring out common terms corresponding to the singular points on the circle of singular foci, one is left with a polynomial of degree 32 and an attempt to use Stürm sequences (see, for example, [12]) proved unsuccessful.

\section{$\S 4$ Kinematic Geometry}

In this section we establish a way of searching among the coupler curves of a given fourbar for those with the maximum number of inflections. The ideas are those of differential kinematic geometry rather than algebraic geometry. Most of what is presented here is well known. However we have not seen the technical details written down elsewhere.

For a smooth (ie suitably many times differentiable) regular parametrised motion of the plane we may identify at each parameter value (in effect, at each instant, if the parameter is time) those points in the plane whose trajectories possess inflections: for a smooth regular plane curve, they are those with vanishing curvature. The motion of a generic four-bar may be regarded as a regular parametrised motion by mapping its configuration space, defined as a real algebraic variety by equations (1) with $w=1$, onto the configuration space for the coupler bar alone, as was done in Donelan [3] and Marsh [8]. That it has a smooth local parametrisation is a simple consequence of the Implicit Function Theorem applied to the equations defining the variety; the parameter may be chosen from among the 
variables $x_{i}, y_{i}$, although in practice it is more usual to employ the angular displacement of one of the moving bars.

Suppose $t$ is a local parameter and a particular coupler point $\mathbf{x}=(x, y)$ has trajectory given by $\gamma_{\mathbf{x}}(t)$. Its curvature is given by

$$
\kappa_{\gamma_{\mathbf{x}}}(t)=\frac{\dot{\gamma}_{\mathbf{x}}(t) \times \ddot{\gamma}_{\mathbf{x}}(t)}{\left\|\dot{\gamma}_{\mathbf{x}}(t)\right\|^{3}}
$$

(where $\mathbf{u} \times \mathbf{v}$ denotes $u_{1} v_{2}-u_{2} v_{1}$ ). Thus $\gamma_{\mathbf{x}}$ is singular or has an inflection if and only if

$$
G(\mathbf{x}, t) \equiv \dot{\gamma}_{\mathbf{x}}(t) \times \ddot{\gamma}_{\mathbf{x}}(t)=0 .
$$

If, at a particular instant $t=t_{0}$, the motion has non-zero rate of rotation then there is a unique instantaneous centre of rotation, whose trajectory has a singularity at $t=t_{0}$. The locus of instantaneous centres is the moving centrode. The points $\mathbf{x}$ satisfying $G\left(\mathbf{x}, t_{0}\right)=0$ form a circle, called the inflection circle, passing through the instantaneous centre and tangent to the moving centrode.

The case in which the rate of rotation vanishes is often overlooked. If it exists, the instantaneous centre of rotation must be at the intersection of the extension of input and output bars (since the joints of the coupler bar move on arcs of circles about the other two joints). Therefore, for generic four-bars, the motion of the coupler bar is instantaneously translatory whenever the input and output bars are parallel.

We can make this precise as follows. Suppose the motion is given as a combination of rotation, represented by the $2 \times 2$ orthogonal matrix $A(t)=\exp \theta(t) J$, where $J=\left(\begin{array}{cc}0 & -1 \\ 1 & 0\end{array}\right)$, and translation, represented by the 2 -vector $\mathbf{a}(t)$. Then $\gamma_{\mathbf{x}}(t)=A(t) \mathbf{x}+\mathbf{a}(t)$. Let us assume that $\theta\left(t_{0}\right)=0$ then, provided $\dot{\theta}\left(t_{0}\right) \neq 0$, the instantaneous centre is $\mathbf{x}=-\left[\dot{A}\left(t_{0}\right)\right]^{-1} \mathbf{a}\left(t_{0}\right)$. Equation (7) assumes the form

$$
G(\mathbf{x}, t) \equiv \dot{\theta}^{3}\|\mathbf{x}\|^{2}+(\ddot{\theta} \dot{\mathbf{a}}-\dot{\theta} \ddot{\mathbf{a}}) \cdot \mathbf{x}-\dot{\theta}^{2} \dot{\mathbf{a}} \times \mathbf{x}+\dot{\mathbf{a}} \times \ddot{\mathbf{a}}=0 .
$$

Both the instantaneous centre and the solution set of (8) are independent of parametrisation, in the sense that they are fixed under a non-singular change of parameter $t \mapsto s(t)$.

In the case $\dot{\theta}\left(t_{0}\right) \neq 0$, equation (8) defines a circle. When $\dot{\theta}\left(t_{0}\right)=0$ but $\ddot{\theta}\left(t_{0}\right) \neq 0$ points $\mathbf{x}$ satisfying (8), whose trajectories necessarily possess inflections at that instant, form a line asymptotic to the moving centrode. For generic four-bars the above condition on the derivatives of $\theta$ necessarily holds [3]. This ensures that such instants $t_{0}$ are isolated.

Solutions of the equation (7) form a smooth surface in $\mathbb{R}^{2} \times \mathbb{R} \cong \mathbb{R}^{3}$. Let $M$ denote the total configuration space of the four-bar (a one-dimensional manifold), for which $t$ is a local parametrisation. We can then construct a two-dimensional submanifold $Q \subseteq \mathbb{R}^{2} \times M$ where $(\mathbf{x}, p) \in Q$ precisely when the coupler curve of the point $\mathbf{x}$ satisfies (7) at configuration $p$ with respect to some (and hence any) local parametrisation $t$ of $M$. 
Now consider the projection

$$
\pi: \mathbb{R}^{2} \times M \rightarrow \mathbb{R}^{2}
$$

onto the first factor. For a fixed configuration $p \in M$, the image under $\pi$ of $Q \cap\left(\mathbb{R}^{2} \times\{p\}\right)$ is the inflection circle or line at that configuration. The set of critical values of the map $\left.\pi\right|_{Q}: Q \rightarrow \mathbb{R}^{2}$ is precisely the envelope $\mathcal{E}$ of the family of curves given locally by $G(\mathbf{x}, t)=0$, or loosely, of the inflection circles, see Figure 2. Note that the envelope is well defined provided $\left(G_{x}, G_{y}\right) \neq(0,0)$ whenever $G=0$. This is certainly the case for generic four-bars since $G=0$ is a non-singular curve for fixed $t$. We can establish two important facts about this construction.

\section{Figure 2. Projection of the Inflection} Circles into the Coupler Plane

Theorem 4.1. Given a coupler point $\mathbf{x} \in \mathbb{R}^{2}$, the coupler curve has an inflection or a singular point in configuration $p \in M$ if and only if $(\mathbf{x}, p) \in Q$. Moreover for $\mathbf{x} \notin \mathcal{E}$ the number of pre-image points of $\mathbf{x}$ under $\pi$ in $Q$ is finite and remains constant over any connected neighbourhood of $\mathbf{x}$ in the complement of $\mathcal{E}$.

Proof. The first part follows from (7). For $\mathbf{x} \notin \mathcal{E}$, it is a regular value of $\pi$. Hence its pre-image in $Q$ is a zero-dimensional manifold whose projection into $M$ is closed. But $M$ is compact so the set is finite. (In fact, we can deduce this from the algebraic geometry of the previous section.) Moreover, by an extension of a theorem of differential topology, see Milnor [9] p8, the number of pre-image points remains constant over any connected neighbourhood of regular values.

Our aim is to construct the envelope $\mathcal{E}$ in the coupler plane and then sample coupler points in connected components of its complement. To achieve this construction we appeal to some further kinematic geometry. The moving Ball curve is defined to be the locus of points whose trajectories are non-singular and have higher inflections in their trajectories. For such a point,

$$
\kappa_{\gamma_{\mathbf{x}}}\left(t_{0}\right)=\dot{\kappa}_{\gamma_{\mathbf{x}}}\left(t_{0}\right)=0
$$

and $\dot{\mathbf{x}}\left(t_{0}\right) \neq 0$.

Theorem 4.2. The envelope $\mathcal{E}$ is the union of the moving centrode and the moving Ball curve.

Proof. The envelope consists of points $\mathbf{x}$ such that in some local parametrisation, there exists $t$ such that $G(\mathbf{x}, t)=\frac{\partial G}{\partial t}(\mathbf{x}, t)=0$. From the definition of $G$,

$$
\frac{\partial G}{\partial t}(\mathbf{x}, t)=\dot{\gamma}_{\mathbf{x}}(t) \times \dddot{\gamma}_{\mathbf{x}}(t)
$$


Hence $\mathbf{x} \in \mathcal{E}$ if and only if $\dot{\gamma}_{\mathbf{x}}(t)=0$ or $\dot{\gamma}_{\mathbf{x}}(t) \times \ddot{\gamma}_{\mathbf{x}}(t)=\dot{\gamma}_{\mathbf{x}}(t) \times \dddot{\gamma}_{\mathbf{x}}(t)=0$, if and only if $\mathbf{x}$ is the instantaneous centre or a Ball point.

The geometry of the moving centrode is well known. It is an algebraic curve of degree eight and is the projection of a variety isomorphic to the configuration space of the four-bar mechanism, Marsh [8]. Hence the number of components of the moving centrode is the same as that of the configuration space. Moreover, as a complex projective variety, the cusp curve has four points of intersection with each endpoint of the coupler bar and four points on the line at infinity. The number of each of these that is real is determined by the Hain class of the mechanism. Corresponding configurations of the four-bar are those in which one pair of moving bars is parallel.

We now give a description of the Ball curve. Müller [11] shows in principle how to obtain an expression for the curve and also obtains a rational relation between its coordinates and those of the centrode. However it is not clear whether the curve is algebraic nor, if so, what its degree would be. We therefore proceed with a topological description.

Theorem 4.3. For almost all four-bars, the Ball curve is a well-defined smooth plane curve with a unique point for each configuration in which the instantaneous centre is finite. It shares the same asymptotes as the moving centrode and has the same number of branches through the endpoints of the coupler bar, the branches being tangential at those points.

Proof. If $\dot{\theta} \neq 0$, then as already noted, the first condition of (8) defines a conic; the second defines a nodal cubic curve (curve of stationary curvature or circling-point curve), with node at the instantaneous centre. Provided the two curves do not share a common component, then by Bézout's Theorem they intersect in 6 points. Two intersections are the isotropic points and a further three occur at the instantaneous centre of rotation. The Ball point at an instant is the remaining point of intersection (which must necessarily be real).

In certain cases this point may coincide with the instantaneous centre; correspondingly, the coupler curve possesses a higher cusp (eg a rhamphoid cusp) [4]. In that case the locus of Ball points osculates the moving centrode. One special instance of this is when the four-bar is in a 'limiting' position, that is when the coupler bar is parallel to one of the input/output bars. Then the instantaneous centre is an endpoint of the coupler bar and it lies at an extremum of its trajectory which is an arc of a circle. It follows that the number of branches of the Ball curve through each of these points is the same as the number of branches of the moving centrode and that these branches are pairwise tangential. This can be seen in Figures 5-8 below. 
The exceptional cases are (i) when $\dot{\theta}=0$, and (ii) when the cubic degenerates to a line and a circle and the latter coincides with the inflection circle.

In case (i), given that $\ddot{\theta} \neq 0$, the curve of stationary curvature degenerates to a conic, there being two subcases $(a)$ a rectangular hyperbola (the line of inflections being an asymptote) or (b) a pair of orthogonal lines (the line of inflections being one of them). So the Ball points are either $(a)$ a point at infinity or $(b)$ a line.

In the case (ii), which is considered in some depth by Veldkamp [14], the Ball points form a circle minus one point (the instantaneous centre).

The two cases (ib) and (ii) in which the Ball points at an instant form a curve are exceptional among four-bars, in the sense that the set of design parameters for which they can occur has codimension one in the open subset $\Gamma$ of the 4-dimensional space of design parameters corresponding to generic mechanisms. In terms of the representation of the motion of the coupler bar by the functions $\theta(t)$ and $\mathbf{a}(t)$, where $t \in I$ is a local parameter, the situation $(\mathrm{i} b)$ requires $\dot{\theta}=0$ and $\dddot{\theta}(\dot{\mathbf{a}} \times \ddot{\mathbf{a}})=\ddot{\theta}(\dot{\mathbf{a}} \times \dddot{\mathbf{a}})$. The first of these determines at most finitely many configurations of any generic four-bar; the second then imposes a condition on the design parameters. Similarly, (ii) requires a pair of conditions. If coordinates are chosen so that the instantaneous centre lies at the origin (whence $\dot{\mathbf{a}}=\mathbf{0}$ ) then the conditions are given vectorially by $\dddot{\mathbf{a}}=\mathbf{0}$.

Precisely, the conditions define codimension 2 submanifolds of the bundle of 3 -jets of planar motions. The map which takes $\left(d_{1}, \ldots, d_{4}, t\right)$ into the bundle is transverse to these submanifolds and so their pre-images are codimension 2 submanifolds of $\Gamma \times I$. The claim follows.

\section{$\S 5$ Computer Graphics}

The realisation of coupler curves with various numbers of inflection points up to the maximum 12 was completed by computer. Initially, the coupler plane of a given four-bar was stratified by means of the envelope of inflection curves. Then for special choices of design parameters we choose coupler points in various components of the stratification.

Rather than trying to construct the various curves algebraically, it was more straightforward to parametrise the configuration space of the four-bar. While the configuration space itself is capable of parametrisation by elliptic functions, it is easier to use piecewise analytic parameters, namely the angle of rotation of one of the moving bars. The problem is simply that of determining the endpoints of the coupler bar. For this purpose the input angle alone is sufficient, for although it does not give a valid parametrisation at limiting positions of the input bar, it is only necessary at such positions to make a sign change in 
the root of a quadratic: one endpoint is uniquely determined and there are at most two choices for the other endpoint. It is necessary to classify the four-bar by Hain type in order to determine the appropriate ranges of input parameter. A constant step size was used in the parametrisation. Although this is not the optimum parametrisation, since the coupler point velocity can become high, it has proved sufficient for our purposes.

The stratification was achieved, via Theorem 4.2, using separate algorithms for the moving centrode and the Ball curve, applied at each parameter value of the configuration space. The algorithms were based on standard geometric constructions. The moving centrode was found by locating the point of intersection of input/output bars. It was necessary to identify parameter values for which the centrode went to infinity. Following the construction of Beyer [1], the Ball point was found as the intersection of the line through the instantaneous centre parallel to the pivot point curve focal axis (the reflection of the pivot point curve guideline in the centrode tangent) and the perpendicular line through the inflection pole (the end of the diameter through the instantaneous centre of the inflection circle, this being found by Bobillier's construction).

\section{Figure $3 . \quad$ Transitions of Coupler Curves}

\section{Figure 4. Arrangement of Acnodes near a Centrode Triple Point}

The following strategy was used in locating coupler curves with the maximum number of inflections. First, by equation (5), the three finite double points must all be acnodes. The full singularity type of a coupler curve is determined by the position of the coupler point in relation to both the moving centrode and the transition curve of Müller [10], consisting for generic four-bars of those coupler points whose trajectories exhibit tacnodes. The relevant transitions are illustrated in Figures 3(a) and (b). It follows that, in the space of design parameters, near a mechanism whose moving centrode possesses a triple point there are mechanisms having coupler curves with any number of acnodes from zero to three (see Figure 4). Whether such curves possess 12 inflections depends also on the number of conjugate bitangents, and hence on the position of the coupler point relative to the Ball curve (the transition across the Ball curve is shown in Figure 3(c)). Only cognate classes $\mathrm{I}^{\prime}$ and II have representatives with triple points on their moving centrodes, so other reasoning is necessary for the classes I and $\mathrm{II}^{\prime}$. A method for constructing appropriate four-bars is given by Beyer [1] and specific design parameters are found in Marsh [8]. We are thus able to establish:

Theorem 5.1. There exist four-bar mechanisms of cognate types $I^{\prime}$ and II possessing 
coupler curves with 12 inflections. Moreover there are open neighbourhoods in the 6dimensional space of design parameters and coupler points in which this holds true.

That the property is an open one follows in part from Theorem 4.2 but can also be established from the fact that the equation of the coupler curve varies continuously in both $d_{1}, \ldots, d_{4}$ and the coordinates $k_{1}, k_{2}$ of the coupler point. Specific examples are given in Table 2. The coordinate frame for the coupler point has been chosen so that one end of the coupler bar (where it adjoins the input arm) is the origin, the positive $k_{1}$-axis lies along the coupler bar which is set to length 1 in these coordinates. (Thus the scale of the coupler coordinates may differ from that of the ambient space.) Note that cognates of the DR mechanism in the Hain classes $\mathrm{CR}_{1}$ and $\mathrm{CR}_{2}$ also exhibit coupler curves with 12 inflections.

Table 2. Four-Bar Coupler Curves with 12 Inflections

\begin{tabular}{lll}
\hline Hain class & $\left(d_{1}, d_{2}, d_{3}, d_{4}\right)$ & $\left(k_{1}, k_{2}\right)$ \\
\hline $\mathrm{R}_{\mathrm{ii}}$ & $(0.48,0.48,0.48,1)$ & $(0.5,-0.882)$ \\
$\mathrm{DR}$ & $(0.85,0.35,0.8,1)$ & $(0.4540,-0.3418)$ \\
\hline
\end{tabular}

Calculation of the curvature of these coupler curves at appropriate configurations reveals 12 sign changes and hence by the Intermediate Value Theorem the presence of 12 inflections. In the case of the DR mechanism these are distributed as 8 on one component and 4 on the other. The corresponding stratifications of the coupler plane and selected coupler curves are shown in Figures 5 and 6. Figure 5 also illustrates some transitions between coupler curves corresponding to different components of the stratification. The position and size of the coupler curve in relation to the mechanism are also illustrated.

Figure 5. STRATificATiON AND Coupler Curves for a $\mathrm{R}_{i i}$ Four-Bar

Figure 6. Stratification AND Coupler Curve for a DR Four-Bar

By pursuing the question of the number of acnodes we are able to establish also:

Theorem 5.2. The maximum number of inflections on a coupler curve of a DL (cognate class $\left.I I^{\prime}\right)$ mechanism is 8 and this number is attainable. 
Proof. We show that no coupler curve can have more than one acnode. The result will then follow from Klein's equation. The moving centrode of any DL mechanism has no real points at infinity and no double points [8]. It therefore consists of two disjoint simple closed curves, each dividing the plane into an exterior and an interior region. Since the centrode is symmetric about the coupler bar, neither curve lies in the interior of the other. It follows from Bobillier's construction that the pole normal points outwards along the two curves and hence coupler curves for points in the interior regions possess one less acnode (and one more crunode) than those in the exterior region.

Figure 7. StratificAtion AND Coupler Curve for a DL Four-Bar

Now consider the endpoints of the coupler bar. These must lie in the exterior region by the above argument. Müller [10] showed that they lie on the transition curve; moreover they are crunodes of the transition curve so that nearby there exist coupler points possessing tacnodes. The transition of singularities along a transversal to the transition curve is from a pair of crunodes through a tacnode to a pair of complex conjugate double points. Since there are altogether only three finite double points on such a coupler curve, at most one can be an acnode. The result now follows from equation (5).

The existence of a coupler curve with 8 inflections is confirmed by the mechanism with design parameters $(1.167,1.16,1.01,1)$ and the coupler point $(-0.33,0.45)$. See Figure 7.

\section{Figure 8. Stratification AND \\ Coupler Curve for a $\mathrm{R}_{o o}$ Four-Bar}

Finally, no specific result concerning the maximum number of inflections for the remaining cognate class I has been obtained. We can say that there exist coupler curves with 8 inflections. An example is shown in Figure 8. The design parameters are $(0.22,1.05,0.21,1)$ and the coupler coordinates are $(-0.17,5.45)$.

\section{$\S 5$ Conclusions}

Application of the equations of Plücker and Klein to the equation of a coupler curve of a hinged planar four-bar linkage has enabled us to deduce that 12 is an upper bound on the number of real inflections on such a curve. This result, though based entirely on classical algebraic geometry, appears to be new in the four-bar literature.

However no direct method presents itself of ascertaining whether coupler curves exist which exhibit 12 inflections. Given that coupler curves depend on six parameters (four design 
parameters and two coupler coordinates), trial and error is unlikely to be successfulindeed we are not aware of anywhere that such a curve is illustrated. A search strategy based on kinematic differential geometry and implemented via a computer graphic routine, together with known results on the existence of tri-cuspidal couplers, enabled us to locate coupler curves with up to 12 inflections for certain double rocker, double crank and crankrocker mechanisms.

We were also able to show that another class of double crank mechanisms, the Hain class DL, does not possess couplers with more than 8 inflections. A number of interesting questions remain concerning real inflections of coupler curves. We do not know whether the remaining Hain class can exhibit more than 8 inflections. It would also be valuable to determine bounds on the regions of design parameters and of coupler points for given four-bars within which the maximum number of inflections occurs.

\section{REFERENCES}

[1] R. Beyer, The Kinematic Synthesis of Mechanisms, Chapman and Hall, London, 1963.

[2] J. L. Coolidge, Algebraic Plane Curves, Clarendon Press, Oxford, 1931.

[3] P. S. Donelan, Generic Properties in Euclidean Kinematics, Acta Applicandae Mathematicae 12 (1988), 265-286.

[4] P. S. Donelan, On the Geometry of Planar Motions, Quarterly J. of Math. Oxford 44 (1993), $165-184$.

[5] C. G. Gibson and P. E. Newstead, On the Geometry of the Planar Four-Bar Mechanism, Acta Applicandae Mathematicae 7 (1986), 113-135.

[6] K. Hain, Das Spektrum des Gelenkvierecks bei veränderlicher Gestell-Länge, VDI Forsch Ing.-Wes. 30 (1964), 33-42.

[7] K. H. Hunt, Kinematic Geometry of Mechanisms, Clarendon Press, Oxford, 1978.

[8] D. Marsh, On the Geometry of Mechanisms, PhD Thesis, Liverpool University, 1987.

[9] J. W. Milnor, Topology from the Differentiable Viewpoint, The University of Virginia Press, Charlottesville, 1965.

[10] R. Müller, Über die Doppelpunkte der Koppelkurve, Z. Math. Phys. 34 (1889), 303-305 \& 372-375, trans. Tesar D. in (Translations of) Papers on Geometrical Theory of Motion applied to Approximate Straight Line Motion, Kansas State University Bulletin, 46 (1962), Special Report No. 21..

[11] R. Müller, Über einige Kurven, die mit der Theorie des ebenen Gelekvierecke in Zusammenhang stehen, Z. Math. Phys. 48 (1903), 224-248, trans. Tesar D. in (Translations of) Papers on Geometrical Theory of Motion applied to Approximate Straight Line Motion, Kansas State University Bulletin, 46 (1962), Special Report No. 21..

[12] T. R. F. Nonweiler, Computational Mathematics: an Introduction to Numerical Approximation, Ellis Horwood, Chichester, 1984.

[13] S. Roberts, On Three-Bar Motion in Plane Space, Proc. London Math. Soc. 7 (1875), $14-23$.

[14] G. R. Veldkamp, Curvature Theory in Plane Kinematics, J B Wolters, Gröningen, 1963. 
Zusammenfassung-Die Gleichungen von Plücker und Klein geben eine obere Grenze von 12 für die Zahl der reellen Wendepunkte einer Gelenkkurve eines ebenen Viergelenkgetriebes. Allgemein liegen die Gelenkpunkte, deren Trajektorien Wendepunkte darstellen, für eine beliebige Konfiguration des Getriebes auf einem Kreis. Die Hüllkurve der Wendepunktkreise ist die Vereinigung der Zentrode und der Ballschen Kurve. Diese Kurven zerlegen die Gelenkebene in zusammenhängende Gebiete mit der Eigenschaft, daß jeder Gelenkpunkt die gleiche Zahl von Wendepunkten auf ihrer Trajektorie hat. Das reduziert das Problem, die Gelenkkurve mit hohen Inflektionszahlen zu finden, zu einer endlichen Suche. Mit Hilfe der Computergrafik können wir dann Gelenkkurven finden, die die maximale Inflektionzahl zeigen.

Department of Mathematics, Victoria University of Wellington, PO Box 600, Wellington, New Zealand

E-mail address: Peter.Donelan@vuw.ac.nz 